\title{
VEHICLE ANTI-THEFT SYSTEM BASED ON AN EMBEDDED PLATFORM
}

\author{
Arun Sasi ${ }^{1}$, Lakshmi R Nair ${ }^{2}$ \\ ${ }^{1,2}$ M.Tech Student, Electrical and Electronics Department, Amrita School of Engineering, Tamil Nadu, India, \\ aruntdpa@gmail.com,lakshmi688@gmail.com
}

\begin{abstract}
In modern day vehicles, vehicle anti-theft system is of prime importance. The vehicle anti-theft system presented here consists of multiple layers of protection with one complementing the other, rather than the conventional anti-theft system where a particular system is only being used. The first layer of protection in the system is a Fingerprint recognition, based on which the doors are opened. The Fingerprint matching is done by utilizing the Minutiae based Fingerprint recognition scheme. Also to prevent thieves from breaking the glass and getting inside the vehicle, vibration sensors are used in all the windows with a threshold level to prevent false alarms. Once inside, the vehicle is turned on only with the mechanical keys along with correct key number entry on the combination keypad present, failing to do so for three successive times will result in vehicle getting immobilized by cutting the fuel supply and an alert message is sent to the mobile number of the owner. Further to prevent the seizure of the vehicle, Tyre pressure sensor is also being used which also alerts the owner through a mobile message. The seized vehicle can be tracked using a GPS tracker which is also being attached. The different layers of protection defined are controlled by an ARM 7 based controller acting as the central node. The whole system was tested using a test set up by mimicking the vehicle door, vehicle immobilizer etc. with equivalent motors whereas Fingerprint data was received from Matlab based GUI application. The experimental results proved the functionality of the anti-theft system in working environment.
\end{abstract}

Index Terms: Vehicle anti-theft, Fingerprint recognition, ARM 7, Immobilizer

$* * *$

\section{INTRODUCTION}

The rapid rate at which vehicle thefts has been increasing across the world has called for increasing thrust in the field of vehicle anti-theft systems. This particularly assumes significance for expensive vehicles and those who go behind even more expensive cosmetic modifications. The vehicle anti-theft system typically performs two functions (1) detecting vehicle theft and preventing false alarms (2) alerting the owner.

The main focus while developing the vehicle anti-theft system was to integrate the above features equally. The most important feature is the vehicle security from theft and it has been ensured by providing three layers of anti-theft protection. First the entry to the vehicle is restricted only to the authorized persons with the help of a Fingerprint Recognizer. The Fingerprint of the owner and other authorized persons are stored into the database beforehand and at the time of entry to the vehicle, scanned fingerprints are being crosschecked with the database [9]. The biometric scheme is used as the primary layer of protection since the chances of it being duplicated are very minimal. Also an additional Vibration Sensor is attached to all windows to prevent the intruder from breaking the vehicle glass and accessing the vehicle.
The next layer of protection is provided by mechanical keys of the car and a combination keypad which immobilizes the engine in case of three wrong entries. The immobilized vehicle can be restarted then only with the help of standard immobilization unlock techniques such as manufacturer transponder systems. The third layer of protection is provided to prevent the vehicle from being seized and is done with the help of Tyre Pressure sensor and a GPS Tracker.

The paper has been organised as follows: Section 1: Introduction. Section 2: Related Works. Section 3: Design Approach. Section 4: Implementation. Section 5: Testing. Section 6: Conclusion.

\section{RELATED WORKS}

The Vehicle Anti-theft Systems has been already discussed a lot in the past. A lot of work has been focussing on the recovering the work vehicle once it is being stolen as in [1] where the GSM messages from the owner can be used to turn off the vehicle as well as find the current location of the stolen vehicle by triangulating from the nearest base station. These are generally referred to as Vehicle Tracking System and are useful in controlling and tracking the current location of a stolen vehicle. Similarly in [3] a Vehicle Tracking System is defined which allows the owner to stop the car from a remote 
location. Also an auto tamper detection technology is also introduced which disables the vehicle in case of tampering in the car security network. The vehicle immobilization scheme is the next biggest security feature that has been added in the recent years.

In [2] the authors implemented a security system based on the car mechanical keys and which immobilizes the vehicle in case of a wrong combination key input inside the vehicle. The authors in [4] define a security system with Face Recognition technology which also helps in recognizing the thief whereas the vehicle security system based on inter vehicle communication is presented in [5]. Also a number of recent anti-theft security systems has been based on RFID tags as explained in [6] where RFID tags are used to control the entry into the vehicle and GSM technology is used to alert owner in case of forceful entry. Also a combination of RFID tags with modern techniques such as Immobilizer as presented in [8]. A system based Smart Card to control the entry into the vehicle based on a PIC microcontroller has been presented by the authors in [7].

The analysis of the related works shows that the security systems presented in the recent years has been focussing on improving the remote control feature of the vehicles. Also the RFID based security systems also found wide acceptance in recent years. However the Vehicle Anti-theft system proposed in this paper differentiates itself from others in that the both vehicle tracking function as well the Anti-theft function has been dealt with in this system. Also Fingerprint recognition scheme is used to control entry to the vehicle in this paper, instead of other biometric techniques such as Face Recognition, since it is both cost effective and efficient.

\section{DESIGN APPROACH}

The design of the Anti-theft system has been done with the aim of making efficient use of all the sensors that are being employed. Also care has been taken to make sure that the functioning of one sensor doesn't affect the performance of another. The design of the system can be viewed as shown in Fig 1 where all the individual modules are connected to a central node and the central node decides on the remedial action to be taken based on different situations. All the sensors and end devices of the anti-theft system are integrated together and connected to a microcontroller, which performs the function of the central node. The microcontroller selected for this application is the LPC 2468 with an ARM 7 core. This is done taking into account the number of peripheral devices to be monitored. A separate TMS320 series DSP Processor is used for Fingerprint Recognition and is interfaced to the LPC 2468. A separate DSP Processor is preferred to a single processor because the Fingerprint Recognition is a highly resource consuming. On the other hand, the central node should be always ready and should not miss any of the interrupts coming from any of the sensors, indicating a security breach. The design of the anti-theft system is explained in detail in the following section.

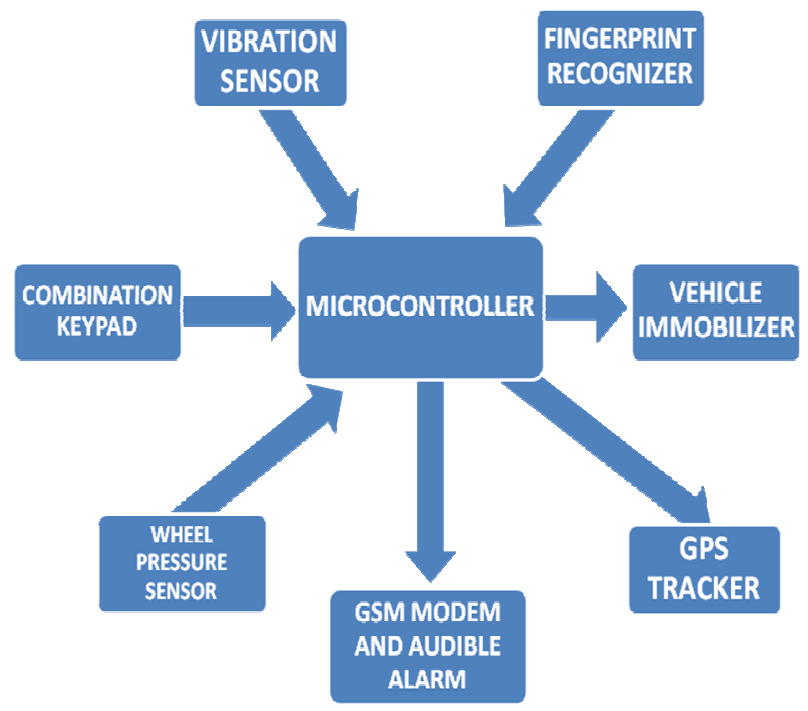

Fig -1: Vehicle Anti-Theft system

\subsection{Multilayered Protection}

The protection of the vehicle has been ensured with the help of a multi-layered architecture. The multi layered architecture is being schematically represented in Fig 2 where each layer is represented in separate blocks. As said earlier, the first layer of protection is offered by the Fingerprint recognition technique. The Fingerprint recognizer is designed to be ideally placed on the door handle of the vehicle and can be used to access the vehicle by the owner. Also a supervisory mode is provided where the Fingerprint of the owner as well as all authorised persons can be stored into the database. The supervisory mode can be accessed only after a valid fingerprint is recognized by the device. The Fingerprint recognizer extracts the minute features of each scan and then it is compared with those of the database. The details of the fingerprint recognition scheme employed are explained later on in the paper. Also to make this layer of protection foolproof, vibration sensors are attached to all window glasses, which detects if the intruder from getting inside by breaking the window glass and alerts the vehicle owner through an attached GSM modem. Thus if the vibration sensor detects a vibration levels greater than the preset threshold limit, alarms are activated and the owner is alerted with the help of the mobile message.

The second layer of protection is being employed with the help of the conventional mechanical key of the vehicle along with the combination key sequence. For that a combination keypad is used which can be ideally placed on the dashboard of the vehicle or any other place which is easily accessible to the driver. Also it is programmed in such a way that if a wrong 
key sequence is detected successively for more than three times, the vehicle will be immobilized. Once immobilized, the immobilization can be restarted only with the help of the vehicle manufacturer. The manufacturer uses transponder or other valid Radio Frequency tags to remove the immobilization. Also a GSM modem is attached to the central node which detects the vehicle immobilization and alerts the driver through a mobile message.

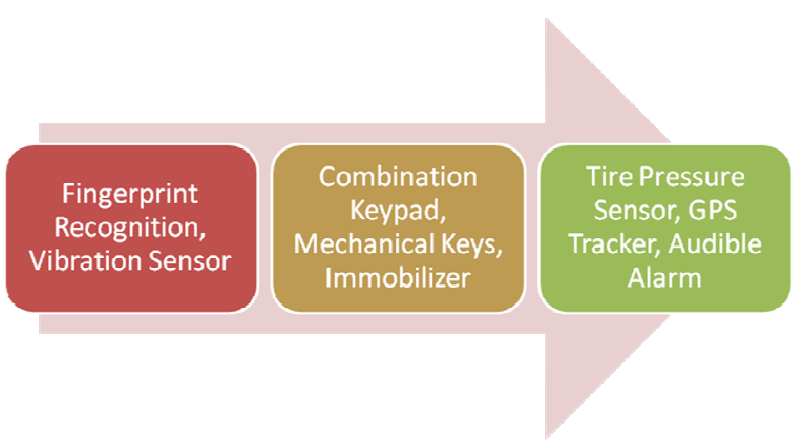

Fig -2: Multilayered Protection

The final protection is provided to prevent the vehicle from being seized by the thief. When the vehicle is parked somewhere there are chances that the vehicle can be seized by the thief in even bigger vehicles such as trucks, so that he can dismantle the vehicle and sell the spare parts. To prevent this Tyre Pressure Sensor is attached to all wheels, so that if the thief tries to lift the vehicle and escape, the sudden change in Tyre pressure will be detected by the sensor and an alert message will be sent to the owner of the vehicle. Also the audible alarms will be activated. Now the vehicle owner can track the current position of his vehicle using the GPS device attached. The GPS provides accurate positions with a precision of few metres and hence the police or the owner can find the current location of his vehicle with ease.

\subsection{Minutiae Based Fingerprint Recognition}

Fingerprint recognition as explained earlier is used to control entry into the vehicle and has been done by employing Minutiae based Fingerprint recognition scheme which is one of the most accurate Fingerprint recognition schemes [9]. Human fingerprints are rich in details called minutiae as shown in Fig 3, where a sample Fingerprint is shown on the left side and Minutiae that is extracted from the sample Fingerprint is shown on the right hand side. Minutia refers to the various terminations and bifurcations on the human Fingerprint pattern, locations of which are unique for each and every individual. To achieve good minutiae extraction in fingerprints with varying quality, pre-processing of the Fingerprint in form of image enhancement and binarization is done on Fingerprints before they are evaluated.

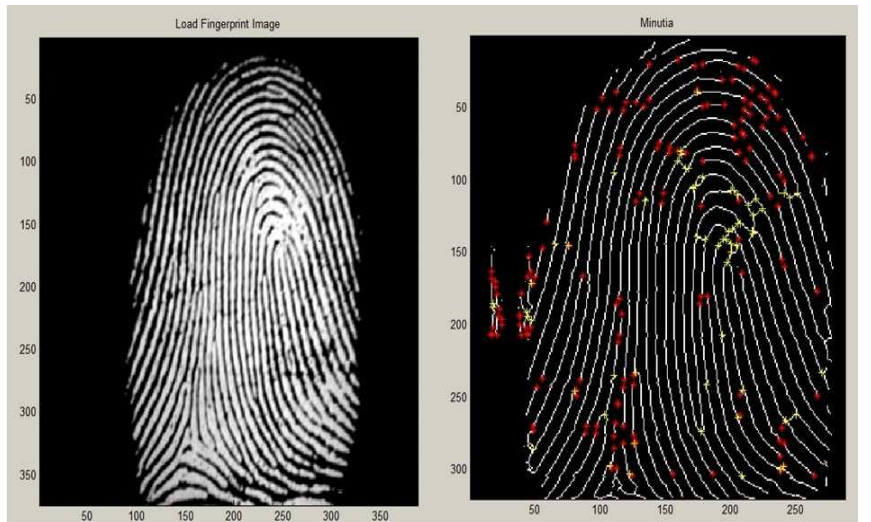

Fig -3: Fingerprint Minutiae

Minutia-marking with false minutia removal methods are used to ensure uniqueness. An alignment-based elastic matching algorithm is used for minutia matching. This technique helps in finding the correspondences between input minutia pattern and the stored template minutia pattern without resorting to an exhaustive search.

\section{IMPLEMENTATION}

The implementation of the Anti-theft system is done by following the design approach as discussed earlier. The Fingerprint recognition algorithm based on the Minutiae based Fingerprint recognition technique is loaded into the DSP Processor. The TMS320x series processor is used and the Matlab code written can be ported on to the processor using Code Composer Studio. The Fingerprint recognizer module is connected onto the TMS320 kit, to deliver the scanned Fingerprints and the Fingerprint Recognizer thus developed is interfaced into the ARM 7. The function of the TMS320 processor is to compare the current Fingerprint with those in the database and thus to confirm the authenticity of the person trying to access the vehicle to the ARM 7 microcontroller. Thus the microcontroller opens the car lock when a valid Fingerprint pattern is recognized by the Fingerprint recognizer kit.

The combination keypad with 10 digit numeric keys is interfaced with LPC 2468 kit and the vehicle immobilization scheme is implemented by accessing the motor which controls the fuel pump in the vehicle. This is achieved by cutting the power relays to the fuel pump, thus preventing the engine from getting enough fuel for its functioning. Also the GSM module is interfaced through the UART port with the default target mobile Sim number of the vehicle owner preset to which the alert messages will be sent. The Vibration Sensor and the Tyre Pressure Sensors are digital and are thus interfaced directly to the ARM 7 microcontroller. The GPS module is also interfaced as defined before. In the event of the vehicle getting lifted up, the Tyre Pressure Sensor detects this and the Microcontroller alerts the vehicle owner through a 
mobile message. Further the GPS tracker is turned on which helps the owner to keep track of the stolen vehicle. In addition to all these features, an audible alarm is also interfaced to the microcontroller which is triggered by in all security breach situations.

\section{TESTING}

Once the implementation of the anti-theft system was done using the various sensors and the LPC 2468 board, the testing of the module was done. The testing of the Anti-theft system was done using a specially made test set up. The Fingerprint Recognizer was tested with the help of a Matlab GUI. A database of Fingerprints was made available. One of the Fingerprints from the database was selected randomly and was assigned as the Fingerprint of the owner and was stored in the LPC 2468 memory. For that, the Fingerprint recognition Algorithm was coded in Matlab initially. Now using the algorithm, the minutiae features of the Fingerprint was extracted. For that the pre-processing was done in Matlab and later the minutiae of the fingerprint were extracted. After that, the matching was done, for that the position and orientation of the newly extracted features were matched against the features of the impressions of the vehicle owner. If the features were matching, the LPC 2468 microcontroller opened the Vehicle lock, which indicated the correct functioning of the primary layer of anti-theft system. This can be done in a vehicle by accessing the motor which controls the car lock. For the purpose of testing, the lock arrangement was set up on the LPC 2468 development board. The lock was simulated with the help of a DC stepper motor with its idle position defined as the locked state. Now if the correct Fingerprint was recognised, the motor will rotate for a predefined amount to time, indicating door unlocking. The test set up developed is shown in Fig 4.

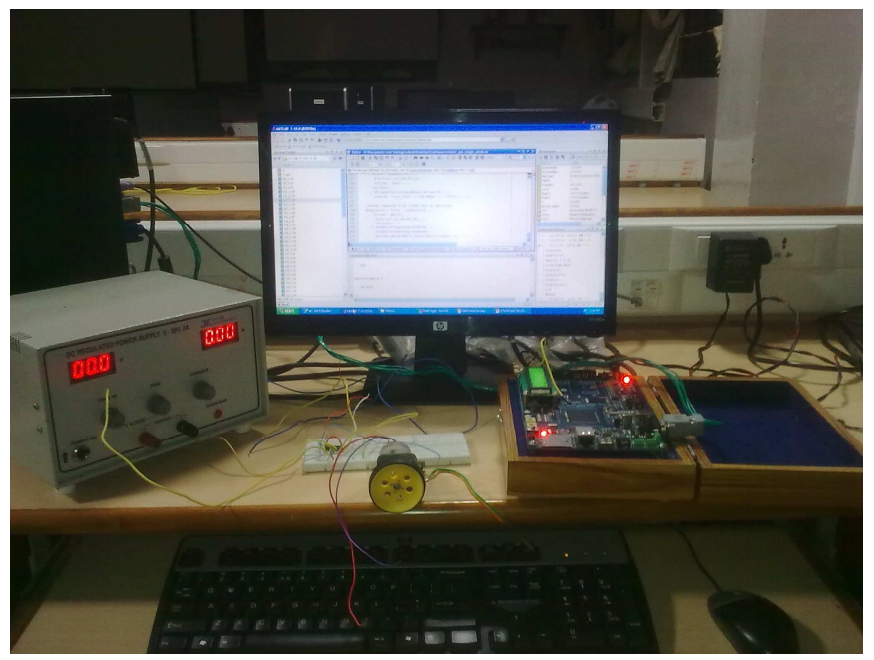

Fig -4: Test Set up
Now the immobilizer can also be implemented using a motor arrangement which was used for testing the previous layer. In an immobilizer, if immobilized the vehicle will not start even if the mechanical keys are inserted and used The motor will be turned on only upon pressing a button switch. However the button switch will be activated only if correct key combination is entered on the keypad provided. The button switch is used to perform the function of the mechanical keys in a typical vehicle. So in this way we can make sure that the car will be turned on only if correct key combination is entered on the keypad along with the mechanical keys. Also the immobilizer section will be turned off if the right matching takes place again. The sensors used in the design were simulated with a set of potentiometers. The GSM module was interfaced to the LPC 2468 and was configured using the AT commands. The normal GSM Sim was inserted inside the module and the mobile number to which the alert message is to be sent is also configured using the AT commands. The alert message is specific for the kind of alert that is required. The alert message in case of a variation in Tyre Pressure will be different from the alert message in case of car immobilization.

The test sequence followed is explained here. Initially the Fingerprint of the owner is loaded into the database. Now the correct sequence is tested, that is the correct Fingerprints are entered and checked whether the algorithm is detecting correctly. After which correct key number is entered and engine turned on by pressing the push button on the LPC2468 kit. The motor turned indicating the correct functionality of the Anti-theft system. Now wrong inputs were given specifically for each and every step. For each wrong entry, the GSM module generated alert messages to the vehicle owner mobile indicating correct functionality. Thus all test conditions were verified.

\section{CONCLUSIONS}

The Vehicle Anti-theft system has been successfully designed and implemented in an embedded system. The design was experimented in a Test set up where the primary and secondary layers of protection were subjected to various tests. The LPC 2468 platform based on an ARM 7 Core has been found to working well and the Minutiae based Fingerprint recognition scheme was found to be found to be ideal for the designed application. The Tyre Pressure Sensor and the Vehicle window Vibration Sensors has to be fine-tuned before practical use, to prevent any false alarms.

\section{ACKNOWLEDGEMENTS}

The authors would like to express sincere gratitude towards Dr. T.N.P Nambiar, Chairperson, EEE Department, Amrita School of Engineering, Coimbatore. Also the authors are also indebted to Mr. R Shanmugha Sundaram, Assistant Professor, EEE Department, Amrita School of Engineering for his constant guidance and support for completing the work. 


\section{REFERENCES}

[1] Ganesh G.S.P, Balaji B and Varadhan T.A.S, "Anti-theft tracking system for Automobiles", IEEE International Conference on Anti-Counterfeiting, Security and Identification (ASID), pp. 17-19, 2011

[2] Sadagopan V.K, Rajendran U and Francis A.J, "Anti-theft Control System design using Embedded System”, IEEE International Conference on Vehicular Electronics and Safety (ICVES), pp. 1-5, 2011

[3]Huaqun Gao, Cheng H.S, Wu Y.D and Venkatasubramanium A.K, "An Automotive Security System for Anti-theft", Eighth International Conference on Networks, pp. 421-426, 2011.

[4] Bhagavathy P, Dhaya R and Devakumar T, "Real-Time car theft decline system using ARM Processor", Third International Conference on Advances in Recent Technologies in Communication and Computing, pp. 101-105, 2011.

[5] Sencun Zhu and Guohong Cao, "SVATS: A Sensor Network-Based Vehicle Anti-theft System", The Twentyseventh International Conference on Computer Communications, 2008.

[6] Ouyang Hongzhi, Wang Xinlin, Zhu Weihua and Li Yuehua, "Design of Auto-guard system based on RFID and Network", International Conference on Electric Information and Control Engineering, pp 1292-1295, 2011.

[7] Muji S.Z.M, Wahab M.H.A, bin Mohd.Zin M.A and Ayob J, "Simulation of Smart Card Interface with PIC for Vehicle Security System", International OCnference on COmputter and Communication Engineering, pp. 878-882, 2008.

[8] Jayendra G, Kumarawadu S and Meegahapola L, "RFID based Anti-theft Auto Security System with Immobilizer", International Conference on Industrial and Information Systems, pp. 441-446, 2007.

[9] Sangram Bana and Dr. Davinder Kaur, "Fingerprint Recognition using Image Segmantation”, International Journal on Advanced Engineering Sciences and Technologies", Vol. No.5, Issue No.1, pp 12-23.

\section{BIOGRAPHIES}

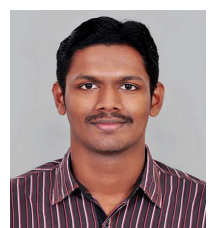

Arun Sasi is an M.Tech Embedded Systems student at Amrita University, Coimbatore. He has completed his B.Tech Degree in Electronics and Communication from Mahatma Gandhi University. His research interests include Sensor Networks, Robotics, Automotive Embedded Systems and Real-Time Systems.

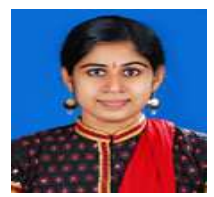

Lakshmi R Nair is an M.Tech Embedded Systems student at Amrita University, Coimbatore. She has completed her B.Tech Degree in Electronics and Communication from Mahatma Gandhi University. Her research interests include Sensor Networks, Digital Signal Processing, Robotics and Automotive Embedded Systems. 